This is an accepted non-edited version of

Reyes Fernandez, B., Warner, L. M., Knoll, N., Montenegro Montenegro, E., \& Schwarzer, R. (2015). Synergistic effects of social support and self-efficacy on dietary motivation predicting fruit and vegetable intake. Appetite, 87, 330-335. doi: 10.1016/j.appet.2014.12.223

\title{
Synergistic effects of social support and self-efficacy on dietary motivation predicting fruit and vegetable intake
}

Background: Self-efficacy and social support are considered relevant predictors of fruit and vegetable intake. This study examines whether the effect of self-efficacy on fruit and vegetables intake is mediated by intention and whether this motivational process is moderated by received dietary social support.

Methods: A longitudinal study with two measurement points in time, four weeks apart, on fruit and vegetable intake was carried out with 473 students aged 19 years on average (52\% women). In a conditional process analysis, dietary intention was specified as a mediator between selfefficacy and fruit and vegetable intake, whereas received dietary support was specified as a moderator of the self-efficacy - intention association, controlling for baseline fruit and vegetable intake.

Results: Self-efficacy was positively associated with fruit and vegetable intake four weeks later, and intention mediated this process. Moreover an interaction between received dietary support and self-efficacy on intention emerged.

Conclusions: The effect of self-efficacy on fruit and vegetable intake was fully mediated by intention. Moreover, received support exhibited a moderating role within the motivational process: high dietary support appeared to accentuate the positive relationship between selfefficacy and dietary intention. 
Synergistic effects of support and self-efficacy on motivation

\title{
Synergistic effects of social support and self-efficacy on dietary motivation predicting fruit and vegetable intake
}

\author{
Consuming at least five portions of fruit and vegetables a day is recommended to prevent
}

chronic diseases and maintain good health (WHO, 2003). However, although this dietary

behavior is widely promoted, breaking unhealthy habits is a difficult self-regulatory task (Slavin

\& Lloyd, 2012). The process of acquiring regular fruit and vegetable intake of about five portions

involves motivational factors that go beyond mere knowledge about nutritional facts. The

formation of an intention is instrumental for the initiation and maintenance of healthy dietary

behaviors, as it sets a self-regulation process in motion that facilitates later goal-relevant

activities (O'Donnell, Greene, \& Blissmer, 2014). The present study, therefore, takes a

longitudinal social-cognitive perspective (Bandura, 1997) to further the understanding of those

psychological mechanisms that underlie healthy eating habits. It is proposed that social exchange

processes interact with motivational factors and hereafter contribute to elicit dietary behaviors.

Although, in most of the health behavior theories, social influences are not explicitly specified as determinants, they are generally considered as being facilitating factors for motivation as well as

for action. Some recent studies have therefore integrated social support into health behavior

change models (e.g. Burkert, Scholz, Gralla, Roigas, \& Knoll, 2011; Ochsner et al., 2014).

However, while the recent literature underscores the facilitating effect of support in the health behavior change process, the underlying mechanisms remain an unresolved issue. A likely mechanism is the synergistic relationship between self-efficacy and social support on motivation and action, which is in line with Bandura's (1997) social cognitive theory (see Warner et al., 2011). The present study follows this line of reasoning and sets out to examine the putative synergistic effect between self-efficacy and the receipt of social support on fruit and vegetable consumption. 


\section{Dietary self-efficacy}

Self-efficacy portrays individuals' beliefs in their capabilities to perform a specific action required to attain a desired outcome (Bandura, 1997). It reflects optimistic self-beliefs when overcoming temptations or adopting a novel course of action. Different challenges could emerge during the course of dietary behavior change, and dietary self-efficacy beliefs may be required to master these tasks successfully. Self-efficacy has been found to be associated with consumption of fruit and vegetables, and individuals with high levels of dietary self-efficacy consume more fruit and vegetables than others (e.g., King et al., 2010).

Evidence for the relevance of self-efficacy for fruit and vegetable intake has also been found in intervention studies, where behavior change techniques led to increases in self-efficacy levels, which were subsequently associated with a more balanced diet (Kreausukon, Gellert, Lippke, \& Schwarzer, 2012; Lhakhang, Godinho, Knoll, \& Schwarzer, 2014; Luszczynska, Tryburcy, \& Schwarzer, 2007).

Self-efficacy is proposed to be relevant in early motivational stages of behavior change, which are directed toward the formation of an intention. In the context of dietary changes, selfefficacy was indeed found to help individuals form dietary goals (e.g., Godinho, Alvarez, Lima, \& Schwarzer, 2014). When individuals are already motivated, self-efficacy gives them the confidence to implement their intentions and initiate and maintain behavioral changes in volitional stages of the change process (e.g., Gutiérrez-Doña, Lippke, Renner, Kwon, \& Schwarzer, 2009). The focus of the present study is on the motivational process, examining how self-efficacy contributes to form intentions directly, whereas dietary behavior would be affected indirectly later on. Put differently, in the present research design, self-efficacy would affect dietary behaviors in two ways: directly as well as indirectly via intention (Ochsner, Scholz, \& 
74 Hornung, 2013). In the present case, intention is specified as the most proximal predictor of

75 behavior.

\section{Received dietary social support}

Social support is a relevant variable in the context of health behaviors, either by directly

78 predicting health behavior (e.g., Darbes \& Lewis, 2005) or by interacting with other health

79 related factors (e.g., Warner, Ziegelmann, Schüz, Wurm, Tesch-Romer, et al., 2011). It refers to

80 the function and quality of social relationships and involves an interactive process between a

81 provider and a receiver. Thus, a distinction can be made between perceived and received social

82 support (Schwarzer \& Knoll, 2010). Perceived social support is the recipient's anticipated

83 available support from its social network if needed. As it is a rather stable cognition, which

84 resembles a personality disposition, it is hardly amenable to interventions (Brand, Lakey, \&

85 Berman, 1995). Received social support, on the other hand, describes the recipient's report about

86 support he or she received in the past. As received support can be prompted directly in

87 interventions, it is a promising factor to be investigated in the nutritional change process (Yates et 88 al., 2012).

Further, it is necessary to measure received social support specifically for the behavior under study. A recent review suggests that general social support is often not related to a healthy diet, whereas received dietary social support has a strong positive link to healthy eating (Tay,

92 Tan, Diener, \& Gonzalez, 2013). Behavior-specific social support may be particularly relevant in

93 the process of goal setting. As pointed out by Scholz, Ochsner, Hornung, and Knoll (2013),

94 received dietary instrumental support predicts dietary intentions.

\section{Individual and social factors for dietary behavior}


Synergistic effects of support and self-efficacy on motivation

Besides its individual regulation, dietary behaviors are often affected by the social context

97 and are therefore expected to be dependent on both self-efficacy and social support according to

98 Bandura's (1997) social-cognitive theory. However, Ochsner et al. (2014) state that research on

99 the interaction between social support and individual regulation of health behavior is still scarce.

The need of studies that investigate the interactions and integration of individual and social factors involved in dietary behaviors is underscored by findings about the psychological resources that best predict fruit and vegetable consumption. In a review conducted by Shaikh,

103 Yaroch, Nebeling, Yeh, and Resnicow (2008), the evidence for the effects of self-efficacy, social

104 support, and knowledge on fruit and vegetable recommendations on consumption were even

105 higher than that of intention. Unfortunately, no distinction was made between perceived and received social support in this review. Previous empirical evidence further suggests a moderating role of received social support on self-efficacy in other health promoting behaviors such as physical activity (Reyes Fernández, Montenegro-Montenegro, Knoll, \& Schwarzer, 2014; Warner, Ziegelmann, Schüz, Wurm, \& Schwarzer, 2011). In the present study, action self-efficacy, received dietary social support, and intention are 111 therefore chosen as predictors for fruit and vegetable consumption. Scholz et al. (2013) proposed 112 to include social support in the motivational phase of the behavior change process, because it was 113 found to contribute to intention formation. Within the context of physical activity it has been 114 found that social support interacts with motivational self-efficacy to increase action control 115 (Reyes Fernández et al., 2014). Besides the volitional contributions of social support reported in 116 previous literature (Ochsner et al., 2014), this suggests a contribution of social support to 117 motivational processes which might set post-intentional processes into motion. 
Synergistic effects of support and self-efficacy on motivation

119

120

121

122

123

124

125

126

127

128

129

130

131

132

133

134

135

136

137

138

139

140

141

142

The aim of the present study is to examine the predictive value of self-efficacy, intention, and social support on fruit and vegetable intake in young adults. Moreover, the question is whether self-efficacy directly relates to fruit and vegetable intake or is mediated via intention and whether there is an interaction between self-efficacy and social support on intention.

We examined these hypotheses:

1. Self-efficacy (T1) predicts fruit and vegetable intake (T2) and intention (T2).

2. Intention (T2) is associated with fruit and vegetable intake (T2) and also mediates the effect of self-efficacy (T1) on fruit and vegetable intake (T2).

3. Received dietary social support (T1) not only predicts intention (T2) but is more strongly associated with intention if self-efficacy (T2) is above average as well - assuming a moderating effect. Put differently, supported individuals build intentions more easily and benefit more from being self-efficacious in terms of their fruit and vegetable intake.

1

\section{Method}

\section{Participants and procedures}

First-year students from a Costa Rican university were invited for a study on healthy life styles in humanities courses in September 2013. In the Costa Rican University system, each student, from any field, has to take courses on philosophy, communication, history and arts, prior to more specialized courses. These courses are offered in several campuses and several buildings all around the country. The research team visited 20 of these classes and invited about 700 students of these introductory lessons at the main Campus in San José. They did not receive credit or financial reimbursement for their participation, but professors encouraged students to take part in the study during the time spans between lessons. After having given informed consent, 663 students filled out a paper and pencil questionnaire at Time 1 (T1, about $95 \%$ of 
Synergistic effects of support and self-efficacy on motivation

143 those invited) and directly returned it to the research team. One month later, in a second visit of

144 the research team to their classrooms, $473(71 \%)$ participants filled out Time 2 (T2)

145 questionnaires. Of those who participated at T2, 52.3\% were women. Their mean age was 18.77

146 years $(S D=2.87)$. Most of the participants were single $(98.2 \%)$, and living in the province of San

147 José $(55.4 \%)$.

$148 \quad$ Measures

149 Self-efficacy was measured at T1 by three items, such as "I am confident that I can eat fruit

150 and vegetables on a regular basis" validated in Lhakhang et al. (2014). Responses could range

151 from 1 (not at all true) to 4 (exactly true) and were averaged.

152 Dietary intention to eat fruit and vegetables were assessed with three items by Lhakhang et

153 al. (2014) at T2. Responses could range from 1 (not at all true) to 4 (exactly true) and were

154 averaged. An example item is "I intend to eat fruit and vegetables every day".

155 Received dietary social support was measured by three items at T1. The original scale is the

validated Family and Friends Support for Heart Healthy Eating Habits Scale by Sallis, Grossman,

157 Pinski, Patterson, and Nader (1987). In this study a three-item short version from Warner,

158 Ziegelmann, Schüz, Wurm, and Schwarzer (2011) was formulated to assess dietary-specific

social support from anyone in the social network and answering categories were adapted to the 4-

160 point Likert scale also used for intentions and self-efficacy. An example item is "There was

161 someone who encouraged me to eat healthily on a regular basis".

Reliabilities for the social-cognitive variables are reported in Table 1.

Fruit and vegetable intake was assessed at baseline and at T2. Two questions, one on the

number of fruit and another on the number of vegetables consumed per day in the past week, 
Synergistic effects of support and self-efficacy on motivation

166 eaten...portions of fruit by day" and "Within the last week, I have eaten...portions of vegetables

167 by day". A portion was defined at the beginning of the questionnaire as the amount of food that

168 fits into one hand. For the analysis, portions of fruit and vegetables were summed up. The

169 internal consistency of this measure was acceptable (Spearman-Brown's $\rho=.63$ ). This instrument

170 has been found to be valid in several studies in different cultural contexts (Kreausukon et al.,

171 2012; Lhakhang et al., 2014; Luszczynska \& Haynes, 2009).

\section{Data analysis}

IBM SPSS 22 was used for the statistical analyses. Drop-out analyses were conducted by means of MANOVA in case of continuous variables, and by means of $\chi^{2}$ in case of categorical variables. Missing values of all variables were below 5\%, and, therefore, listwise deletion was 176 justified.

To examine the first two study hypotheses, a simple mediation model was carried out by means of the SPSS Process macro by Hayes (2012). In a first step, intention as putative mediator

179 (T2) is regressed on self-efficacy (T1), which is here an independent variable. As a second step, the dependent variable (T2 fruit and vegetable intake) is regressed on the independent variable self-efficacy (T1), on the putative mediator intention (T2) and on covariates:T1 fruit and vegetable intake. All paths were estimated by means of ordinary least squares procedures.

183 Confidence intervals (95\%) were generated by bootstrapping with 5,000 resamples. Bootstrapped 184 confidence intervals do not require a normal distribution and are therefore recommended when 185 indirect effects are examined (Hayes, 2013).

186 Once the simple mediation was corroborated, a conditional process analysis was conducted.

187 Conditional process models are those which integrate mediation and moderation analyses (Hayes, 188 2013). In this case, in accordance with the third hypothesis, a moderation between the 
Synergistic effects of support and self-efficacy on motivation

189 independent variable and the putative mediator was expected. Thus, intention (T2) was regressed 190 on self-efficacy (T1) and social support (T1) as well as on the interaction term of self-efficacy

191 (T1) with social support (T1). Thereafter, fruit and vegetable consumption (T2) was regressed on

192 self-efficacy (T1), intention (T2) and baseline fruit and vegetable consumption as covariate.

193 Bootstrapped $95 \%$ confidence intervals (5,000 resamples) were generated. In this conditional

194 process analyses an estimate for the indirect effect was generated, as well as estimates at different

195 levels of the moderator.

196

197

Results

\section{Drop-out analysis}

There was a drop-out rate of $28.7 \%$ (completers $=473$, non-completers $=190)$. The main

reason for drop-out was that $\mathrm{T} 1$ participants were not encountered in their respective $\mathrm{T} 2$ courses.

201 No differences were found between completers and non-completers regarding sex, self-efficacy,

202 social support, and baseline fruit and vegetable intake. However, there was a small difference in

203 age: those who dropped out were older than those who completed T2 $\left(M_{\text {completers }}=18.78\right.$ years,

$204 S D_{\text {completers }}=2.87 ; M_{\text {non-completers }}=19.30$ years, $S D_{\text {non-completers }}=3.26 ;$ Cohen's d $=0.17$ ).

205

206

Insert Figure 1 about here

207

Descriptive statistics

Table 1 shows the means, standard deviations, and internal consistencies for each study

210 variable, as well as the intercorrelations among them. As can be seen, about six portions of fruit

211 and vegetables were consumed daily at baseline and one month later. All study variables were

212 closely associated with each other. The highest correlation was between baseline and subsequent 
213 fruit and vegetable intake at T2 $(r=.53)$, suggesting high rank stability of this behavior across

214 the two measurement points. The correlation between self-efficacy (T1) and intention (T2) with $r$

$215=.50$ can as well be classified as large in accordance with Cohen's (1988) guidelines. The

216 association of social support with dietary intention $(r=.28)$ as well as the association between

217 self-efficacy and behavior $(r=.28)$ showed a medium effect size.

\section{Mediation analysis}

219 Dietary intention was specified as a mediator between dietary self-efficacy and T2 fruit and 220 vegetable consumption, controlling for baseline behavior. In the first mediation step, self-efficacy 221 predicted intention $(b=.60, \mathrm{SE}=.04,95 \% \mathrm{CI}[0.51,0.69], p<.001)$. In the second analysis step, 222 intention $(b=.47, \mathrm{SE}=.22,95 \% \mathrm{CI}[.0 .02,0.90], p=.03)$ and baseline fruit and vegetables 223 intake $(b=.52, \mathrm{SE}=.04,95 \% \mathrm{CI}[0.44, .61], p<.001)$ had effects on T2 fruit and vegetable 224 intake, but not self-efficacy $(b=.22, \mathrm{SE}=.27,95 \% \mathrm{CI}[-0.30,0.75], p=.39)$. The indirect effect 225 of self-efficacy on behavior was $b=.28,95 \%$ CI $[0.03, .56]$. The total effect of self-efficacy on 226 fruit and vegetable intake was $b=.49,95 \%$ CI $[0.03,0.95], p=.04$. The simple mediation model 227 specified accounted for $R^{2}=.29$ of the variance in fruit and vegetable intake.

To further examine the putative effects of social support on dietary intention, T1 support was specified as a moderator between T1 self-efficacy and T2 intention.

Figure 1 shows the main results. In the first step, intention (T2) was regressed on self- 
236 Intention was predicted by self-efficacy $(b=.33, \mathrm{SE}=.11,95 \% \mathrm{CI}[0.10,0.55], p<.01)$, by the

237 interaction of social support with self-efficacy $(b=.10, \mathrm{SE}=.04,95 \% \mathrm{CI}[0.01,0.20], p=.02)$,

238 but not directly by social support $(b=-26, \mathrm{SE}=.16,95 \%$ CI $[-0.57,0.09], p=.15)$. The total

239 effect of social support on intention $(r=.28, b=.23)$ may have been suppressed by self-efficacy

240 and the interaction term of self-efficacy and social support. In a second step, fruit and vegetable

241 intake (T2) was regressed on self-efficacy (T1), intention (T2), and baseline fruit and vegetable

242 intake as a covariate. Dietary intention $(b=.46, \mathrm{SE}=.22,95 \% \mathrm{CI}[0.02,0.90], p=.04)$ and

243 baseline behavior $(b=.52, \mathrm{SE}=.04,95 \% \mathrm{CI}[0.44,0.61], p<.001)$ showed significant direct

244 paths toward T2 fruit and vegetable intake, but not self-efficacy $(b=.22, \mathrm{SE}=.27,95 \% \mathrm{CI}[-$

$2450.30,0.75], p=.39)$. The path from self-efficacy to behavior was mediated via intention with an

246 indirect effect of $b=.28$, bootstrapped $\mathrm{SE}=.13,95 \%$ bootstrapped CI [0.02, 0.55], when

247 received social support is at the mean. The indirect effect of self-efficacy on behavior was higher

248 among those participants with $1 S D$ social support above the mean $(b=.33$, bootstrapped $\mathrm{SE}=$

$249.16,95 \%$ bootstrapped CI $[0.03,0.66])$ than among those with $1 S D$ social support below the

250 mean $(b=.23, \mathrm{SE}=.11,95 \%$ bootstrapped CI $[0.02,0.48])$. The positive relationship between

251 self-efficacy and intention is hence stronger among individuals with higher levels of received

252 social support $(b=.10, \mathrm{SE}=.04,95 \% \mathrm{CI}[0.01,0.20], p=.02)$ as illustrated in Figure 2.

253 Intention was a stronger predictor for T2 fruit and vegetable intake than self-efficacy.

254 Overall, the variables of the conditional process model accounted for $R^{2}=.29$ of the variance in

255 fruit and vegetable intake. 


\section{Discussion}

This longitudinal study explored the synergistic effect of dietary self-efficacy and received 260 dietary social support on the intention to consume fruit and vegetables in young adults. A recent review suggests that self-efficacy, social support, and knowledge are the best predictors of fruit and vegetable consumption, whereas weaker evidence has been found for intention (Shaikh et al., 2008). In many health behavior change theories, the interplay between social and individual

264 factors is often not clearly specified. Bandura's social-cognitive theory (1997) comprises social 265 support as a socio-structural facilitator, which potentially moderates the effects of self-efficacy 266 (Warner, Ziegelmann, Schüz, Wurm, \& Schwarzer, 2011). Given recent evidence on the role of 267 received social support to prompt self-regulatory strategies within the motivation process for 268 physical activity (Reyes Fernández et al., 2014), this study aimed to examine the moderating role 269 of received dietary social support in the dietary motivation process and its subsequent effect on 270 fruit and vegetable intake.

271 Our results confirm most of the hypotheses proposed: On the level of zero-order 272 correlations, self-efficacy at T1 was positively associated with fruit and vegetable intake four 273 weeks later as well as with T2 dietary intention. In other words, self-efficacy contributed to the 274 definition of a dietary goal as well as to the performance of the corresponding behavior at $\mathrm{T} 2$, as 275 formulated in Hypothesis 1.

276 Intention at T2 was also found to be associated with fruit and vegetable intake and 277 mediated the effect of T1 self-efficacy on T2 behavior, supporting Hypothesis 2. Including 278 intention into the model reduced the formerly significant bivariate association between self279 efficacy T1 and T2 fruit and vegetable consumption to zero. 
Synergistic effects of support and self-efficacy on motivation

In bivariate zero-order correlations, received dietary social support had a moderate but

281

282

283

284

285

286

287

288

289

290

291

292

293

294

295

296

297

298

299

300

301

302

positive association with intention. However, when self-efficacy was simultaneously included as

a predictor of dietary intention, the effect of social support was suppressed. Nevertheless, an interaction between self-efficacy and received support on intention was found. Among individuals who received more support for a healthy diet, the association between self-efficacy and intention was stronger, and setting a dietary goal was easier. These results suggest that there may not be a direct effect of received dietary social support on intention, but that the contribution of receiving social support for ones diet in the goal setting process is a moderating one. Thus, the third hypothesis was partially confirmed.

\section{Implications}

When conducting motivational interventions on dietary behavior, techniques to increase self-efficacy, such as providing behavioral role models, giving the opportunity for mastery experience in healthy cooking and eating, or raising the affective value of healthy foods, should be taken into account. In order to make motivational interventions more effective, strategies to increase received dietary social support, such as encouragement or conjoint healthy cooking, might be successful. When social support is the focus of a dietary intervention, it should, however, be kept in mind that support can also be perceived as controlling or overprotecting and needs to be provided in a sensitive way (e.g., Silverman, Hecht, \& McMillin, 2002). Because previous research has found that the interaction between self-efficacy and social support is associated with other health behaviors and self-regulatory strategies (Reyes Fernández et al., 2014; Warner, Ziegelmann, Schüz, Wurm, \& Schwarzer, 2011), future research should be conducted in order to further examine their synergistic effects on post-motivational selfregulatory processes. 
303

\section{Limitations and strengths}

Causal inferences should not be made, because the results of this study are not based upon an experimental design. However, the longitudinal placement of variables and accordance of findings with our theoretical backdrop may support directional conclusions.

The use of self-reports for assessing behavior can be criticized as well, because people may commit unintentional recall mistakes when estimating their past fruit and vegetable consumption or cover up poor eating habits. Future studies should validate such reports against objective data. Further, it would have been desirable to have additional measurement points in time to follow up on the development of social-cognitive variables as well as behavioral changes for an extended period of time.

Despite these limitations, the findings reveal an interplay of motivational factors with the receipt of social support when it comes to the understanding of fruit and vegetable intake among younger Latin American adults. Dietary behaviors may have a strong social dimension, because eating is usually done in a social context. The findings are also consistent with previous literature that has identified associations between self-efficacy, self-reported fruit and vegetable intake, and body mass index, underscoring the importance of self-efficacy for health outcomes (Luszczynska \& Haynes, 2009). Our conclusions constitute a contribution to an understanding of the interplay between social resources and self-regulation in healthy nutrition that can guide future intervention studies. 
Synergistic effects of support and self-efficacy on motivation

324

325

326

327

328

329

330

331

332

333

334

335

336

337

338

339

340

341

342

343

344

345

\section{References}

Bandura, A. (1997). Self-efficacy: The exercise of control. New York: Freeman.

Brand, E. F., Lakey, B., \& Berman, S. (1995). A preventive, psychoeducational approach to increase perceived social support. American Journal of Community Psychology, 23(1), 117-135. doi: 10.1007/Bf02506925

Burkert, S., Scholz, U., Gralla, O., Roigas, J., \& Knoll, N. (2011). Dyadic planning of health-behavior change after prostatectomy: A randomized-controlled planning intervention. Social Science \& Medicine, 73(5), 783-792. doi: 10.1016/j.socscimed.2011.06.016

Cohen, J. (1988). Statistical power analysis for the behavioral sciences (2nd ed.). Hillsdale, N.J.: L. Erlbaum Associates.

Darbes, L. A., \& Lewis, M. A. (2005). HIV-specific social support predicts less sexual risk behavior in gay male couples. Health Psychology, 24(6), 617-622. doi: 10.1037/0278-6133.24.6.617

Godinho, C. A., Alvarez, M.-J., Lima, M. L., \& Schwarzer, R. (2014). Will is not enough: Coping planning and action control as mediators in the prediction of fruit and vegetable intake. British Journal of Health Psychology, 19(4), 856 - 870. doi: 10.1111/bjhp.12084

Gutiérrez-Doña, B., Lippke, S., Renner, B., Kwon, S., \& Schwarzer, R. (2009). Self-efficacy and planning predict dietary behaviors in Costa Rican and South Korean women: two moderated mediation analyses. Applied Psychology: Health and Well-Being, 1(1), 91-104. doi: 10.1111/j.17580854.2009.01004.x

Hayes, A. F. (2012). PROCESS: a versatile computational tool for observed variable mediation, moderation, and conditional process modeling [White paper].

Hayes, A. F. (2013). Introduction to mediation, moderation, and conditional process analysis: a regression-based approach. New York: Guilford Press.

King, D. K., Glasgow, R. E., Toobert, D. J., Strycker, L. A., Estabrooks, P. A., Osuna, D., \& Faber, A. J. (2010). Self-efficacy, problem solving, and social-environmental support are associated with diabetes self-management behaviors. Diabetes Care, 33(4), 751-753. doi: 10.2337/dc09-1746 
Synergistic effects of support and self-efficacy on motivation

Kreausukon, P., Gellert, P., Lippke, S., \& Schwarzer, R. (2012). Planning and self-efficacy can increase fruit and vegetable consumption: a randomized controlled trial. Journal of Behavioral Medicine, 35(4), 443-451. doi: 10.1007/s10865-011-9373-1

Lhakhang, P., Godinho, C., Knoll, N., \& Schwarzer, R. (2014). A brief intervention increases fruit and vegetable intake. A comparison of two intervention sequences. Appetite, 82C, 103-110. doi: 10.1016/j.appet.2014.07.014

Luszczynska, A., \& Haynes, C. (2009). Changing nutrition, physical activity and body weight among student nurses and midwives effects of a planning intervention and self-efficacy beliefs. Journal of Health Psychology, 14(8), 1075-1084. doi: 10.1177/1359105309342290

Luszczynska, A., Tryburcy, M., \& Schwarzer, R. (2007). Improving fruit and vegetable consumption: a self-efficacy intervention compared with a combined self-efficacy and planning intervention. Health Education Research, 22(5), 630-638. doi: 10.1093/Her/Cyl133

O'Donnell, S., Greene, G. W., \& Blissmer, B. (2014). The effect of goal setting on fruit and vegetable consumption and physical activity level in a web-based intervention. Journal of Nutrition Education and Behavior(0). doi: 10.1016/j.jneb.2014.03.005

Ochsner, S., Luszczynska, A., Stadler, G., Knoll, N., Hornung, R., \& Scholz, U. (2014). The interplay of received social support and self-regulatory factors in smoking cessation. Psychology \& Health, 29(1), 16-31. doi: 10.1080/08870446.2013.818674

Ochsner, S., Scholz, U., \& Hornung, R. (2013). Testing phase-specific self-efficacy beliefs in the context of dietary behaviour change. Applied Psychology: Health and Well-Being, 5(1), 99-117. doi: 10.1111/j.1758-0854.2012.01079.x

Reyes Fernández, B., Montenegro-Montenegro, E., Knoll, N., \& Schwarzer, R. (2014). Self-efficacy, action control, and social support explain physical activity changes among Costa Rican older adults. Journal of Physical Activity \& Health. doi: 10.1123/jpah.2013-0175 
Synergistic effects of support and self-efficacy on motivation

374

375

376

377

Sallis, J. F., Grossman, R. M., Pinski, R. B., Patterson, T. L., \& Nader, P. R. (1987). The development of scales to measure social support for diet and exercise behaviors. Preventive Medicine, 16(6), 825836. doi: 10.1016/0091-7435(87)90022-3

Scholz, U., Ochsner, S., Hornung, R., \& Knoll, N. (2013). Does social support really help to eat a low-fat diet? Main effects and gender differences of received social support within the Health Action Process Approach. Applied Psychology-Health and Well Being, 5(2), 270-290. doi: 10.1111/Aphw.12010

Schwarzer, R., \& Knoll, N. (2010). Social support. In J. W. A. Kaptein \& J. Weinman (Eds.), Health Psychology (pp. 283-293). Oxford: Blackwell.

Shaikh, A. R., Yaroch, A. L., Nebeling, L., Yeh, M. C., \& Resnicow, K. (2008). Psychosocial predictors of fruit and vegetable consumption in adults - A review of the literature. American Journal of Preventive Medicine, 34(6), 535-543. doi: 10.1016/j.amepre.2007.12.028

Silverman, P., Hecht, L., \& McMillin, J. D. (2002). Social support and dietary change among older adults. Ageing and Society, 22(1), 29-59. doi: 10.1017/S0144686x02008590

Slavin, J. L., \& Lloyd, B. (2012). Health benefits of fruits and vegetables. Advances in Nutrition, 3(4), 506-516. doi: 10.3945/an.112.002154

Tay, L., Tan, K., Diener, E., \& Gonzalez, E. (2013). Social relations, health behaviors, and health outcomes: a survey and synthesis. Applied Psychology: Health and Well-Being, 5(1), 28-78. doi: 10.1111/Aphw.12000

Warner, L. M., Ziegelmann, J. P., Schüz, B., Wurm, S., \& Schwarzer, R. (2011). Synergistic effect of social support and self-efficacy on physical exercise in older adults. Journal of Aging and Physical Activity, 19(3), 249-261.

Warner, L. M., Ziegelmann, J. P., Schüz, B., Wurm, S., Tesch-Romer, C., \& Schwarzer, R. (2011). Maintaining autonomy despite multimorbidity: self-efficacy and the two faces of social support. European Journal of Ageing, 8(1), 3-12. doi: 10.1007/s10433-011-0176-6 

(2012). The influence of cognitive-perceptual variables on patterns of change over time in rural midlife and older women's healthy eating. Social Science \& Medicine, 75(4), 659-667. doi:

\section{Yates, B. C., Pullen, C. H., Santo, J. B., B
(2012). The influence of cognitive-p
midlife and older women's healthy
10.1016/j.socscimed.2012.01.001}

WHO. (2003). Diet, nutrition and the prevention of chronic diseases - Introduction. Diet, Nutrition and the Prevention of Chronic Diseases, 916, 1-149.

Table 1. Descriptive statistics, reliability, and intercorrelations for dietary self-efficacy, received dietary social support, dietary intention, fruit and vegetable intake.

\begin{tabular}{|c|c|c|c|c|c|}
\hline Variables & 1 & 2 & 3 & 4 & 5 \\
\hline Cronbach's $\alpha$ & .89 & .88 & .96 & - & - \\
\hline Mean & 3.40 & 2.56 & 3.17 & 5.87 & 6.16 \\
\hline Standard deviation & 0.77 & 1.04 & 0.89 & 4.05 & 4.31 \\
\hline 1 Dietary self-efficacy at $\mathrm{T} 1$ & 1 & & & & \\
\hline 2 Received dietary social support at T1 & $.28^{* * *}$ & 1 & & & \\
\hline 3 Dietary intention at $\mathrm{T} 2$ & $.50^{* * * *}$ & $.28^{* * * *}$ & 1 & & \\
\hline 4 Fruit and vegetable intake at $\mathrm{T} 1$ & $.29^{* * *}$ & $.14^{* *}$ & $.24^{* * *}$ & 1 & \\
\hline 5 Fruit and vegetable intake at $\mathrm{T} 2$ & $.23^{* * *}$ & $.10^{*}$ & $.23^{* * *}$ & $.53^{* * *}$ & 1 \\
\hline
\end{tabular}

Note. $* * * p<.001 ; * * p<.01 ; * p<.05$ 


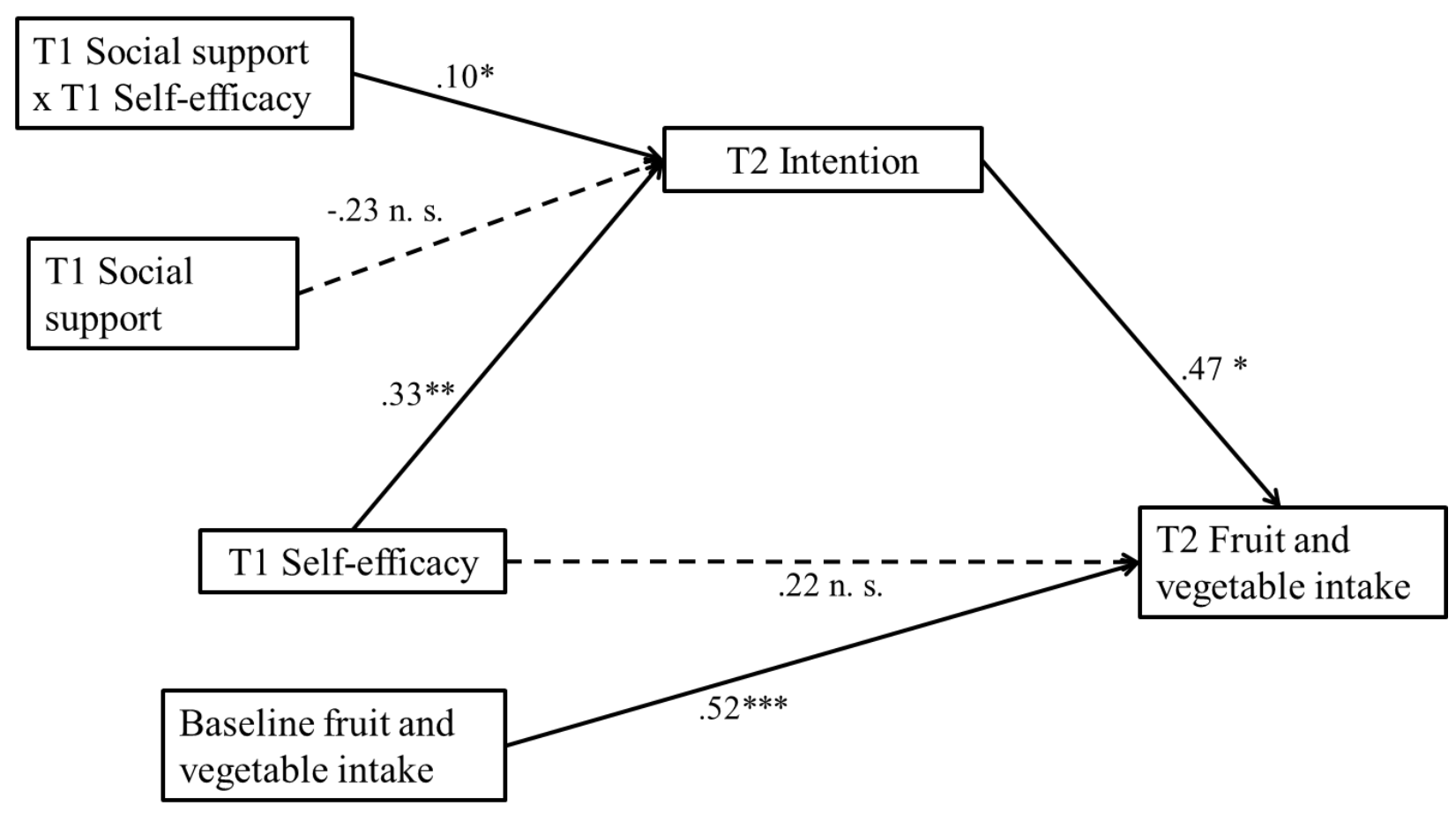

416 Figure 1. Conditional process model to predict changes in fruit and vegetable intake by 417 intention, self-efficacy, and received dietary social support, controlling for baseline behavior.

418 Note. $* * * p<.001 ; * * p<.01 ; * p<.05 .{ }^{\dagger} p=.06$. Parameters are unstandardized $(N=450)$. 


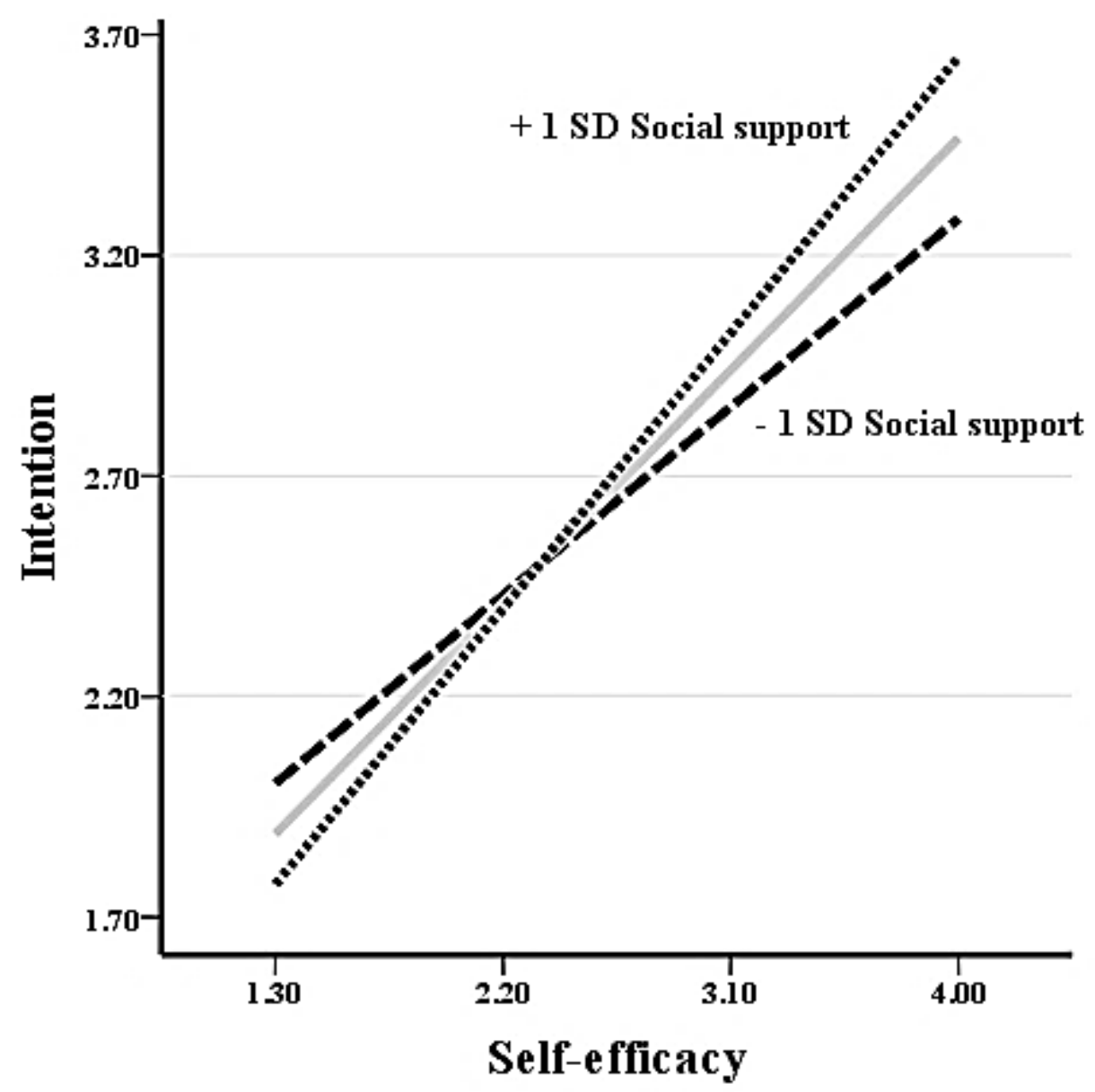

425 Figure 2. Interaction of dietary self-efficacy and received dietary social support on dietary 426 intention. Note: $\mathrm{SD}=$ standard deviation 